\title{
Physical training as a therapeutic measure in chronic heart failure: time for recommendations
}

Over the past decade, traditional medical teaching and advice regarding physical activity in patients with chronic cardiac disease have changed. It is now believed that bed rest and exercise restriction may lead to deconditioning and increased morbidity. Recent studies dealing with both asymptomatic patients with left ventricular dysfunction and patients with symptomatic heart failure support this concept. This has been incorporated in medical knowledge, but so far only marginally applied in clinical practice.

\section{The rationale}

Let us briefly review the rationale of this concept. In normal conditions, ${ }^{1}$ when dynamic exercise starts, signals generated by subthalamic neurons, the so-called central command, determine vagal withdrawal with an immediate increase in heart rate and cardiac output. Sympathetic nerve activity begins to increase later on, when the heart rate approaches 100 beats/min and vagal withdrawal is already almost complete. Central command has little direct influence on the sympathetic nervous system, which mostly responds to chemoreflex, mechanoreflex, and baroreflex signals through the release of several mediators, either endothelial generated mediators (mainly nitric oxide), or myocyte generated metabolites (such as adenosine), which regulate the tone of vessels in active muscles. The short life of these substances allows an instantaneous regulation of the muscle flow according to the rapidly changing metabolic needs of the muscles. ${ }^{2}$ Thus, in daily life activity central command and parasympathetic modulation prevail in regulating cardiovascular function; during physical exercise, sympathetic activity dominates the scene. The limitations of exercise capacity in normal subjects are determined by both the ability to increase cardiac output and the ability of (usually deconditioned) skeletal muscle to perform aerobic activity.

In the heart failure syndrome, two of the main symptoms are fatigue and limitation in exercise capacity. It was assumed that these were caused by the impairment of the mechanical performance of the heart. However, several lines of reasoning led to the conclusion that the generation of symptoms that limit exercise cannot be explained entirely by the haemodynamic status of the patients. Accordingly, much work was concentrated on abnormalities in peripheral blood flow, endothelial function, skeletal muscle, and lung function in chronic heart failure. It has been known for many years that patients with heart failure exhibit subnormal peripheral blood flow response to both exercise induced and pharmacologically induced vasodilatation..$^{-5}$ However, several well treated heart failure patients have greatly reduced exercise tolerance and evidence of early muscular lactate release despite normal skeletal muscle blood flow. All these findings indicate that in many heart failure patients, an inherent defect in skeletal muscle metabolism is operative.

In fact pronounced structural and functional abnormalities in skeletal muscle have been observed in patients with chronic heart failure regardless of aetiology. ${ }^{6-8}$ These include alterations in mitochondria, and in fibre dimension and prevalence, defects in oxidative and glycolytic enzymes which lead to early dependence on the anaerobic metabolism, excessive early depletion of high energy phosphate bonds, and excessive early intramuscular acidification, together with defects in $\beta$ oxidation of fatty acids and an increase in amino acids utilisation. These alterations can lead to early fatiguability and a reduction in maximal muscle strength. In fact, a good correlation exists between defects in skeletal muscle and objective exercise limitations in patients with heart failure. Some of the muscular alterations are similar to those observed in severe deconditioning, while others are more specific to the heart failure condition.

Similar findings were reported in studies dealing with the respiratory musculature. ${ }^{9-11}$ The main results of these studies provided evidence of histochemical abnormalities similar to those observed in limb muscles, reduced respiratory muscle endurance (assessed by maximal sustainable ventilatory capacity), and reduced inspiratory and expiratory forces. A progressive respiratory muscle deoxygenation (assessed by near infrared spectroscopy) has been observed during exercise, indicating early anaerobic metabolism. In spite of this, one of the most relevant respiratory findings in patients with heart failure is excessive ventilation both at rest and during exercise, even when correction for the rate of carbon dioxide production is made.

As a result of these multiple and apparently heterogeneous findings, a "muscle hypothesis" was proposed by Coats and colleagues as the basis of the generation of the exercise intolerance in chronic heart failure. ${ }^{12}{ }^{13}$ According to this hypothesis, the metabolic state of skeletal muscle is centrally monitored by the activation of ergoreceptors, whose fibres, travelling in the lateral spinothalamic tract, increase ventilation and activate the sympathetic system. A reduction in left ventricular function triggers a series of metabolic events that lead to wasting of skeletal muscle and resultant abnormalities of muscle metabolism and function. In response to early metabolic distress in exercising muscle, an exaggerated ergoreflex activation occurs that is perceived by the patient as both muscle fatigue and dyspnoea, and that leads reflexly to excessive sympathetic vasoconstrictor drive to non-exercising muscles and an excessive ventilatory response to exercise. These heartmuscles-brain feedback mechanisms occur in a complex context, because besides inactivity and malnutrition, a complex "catabolic state" with multiple components such as sympathetic activation, insulin resistance, cytoxine production, and other mechanisms generate a true "myopathy" in patients with chronic heart failure. Accordingly, symptomatic improvements of heart failure patients will depend on the resolution of skeletal muscle abnormalities, and will be associated with treatments aimed at specifically improving muscle function or exercise responses. Physical training should be considered one of these. 
Table 1 Summary of randomised exercise training studies in left ventricular dysfunction and heart failure

\begin{tabular}{|c|c|c|c|}
\hline Study & Number of patients & Clinical characteristics & Ejection fraction $(\%)^{*}$ \\
\hline Coats 1990 & 11 & Stable CHF, IHD, sinus rhythm, NYHA II/III & $18(5)$ \\
\hline Jette 1991 & 18 & $\begin{array}{l}10 \text { weeks after first anterior MI, no previous history of heart failure, } \\
\text { NYHA I/II }\end{array}$ & $24(3.5)$ \\
\hline Koch 1992 & 25 & Stable CHF, IHD or IDCM, NYHA II/III & $26(10)$ \\
\hline Meyer 1991 & 12 & Stable CHF, sinus rhythm, NYHA II/III & $23(3.2)$ \\
\hline Coats 1992 & 17 & Stble CHF secondary to IHD, sinus rhythm, NYHA II/III & $20(2.3)$ \\
\hline Davey 1992 & 22 & Stable CHF, NYHA II/III & $22(8.0)$ \\
\hline EAMI study, Gianuzzi 1993 & 31 & 5 weeks after first anterior MI, NYHA II/III & $34(5)$ \\
\hline Adamopoulus 1993 & 12 & Stable CHF, NYHA II/III & $24(3.4)$ \\
\hline Belardinelli 1995 & 27 & Mild CHF, IHD or IDCM, NYHA I/II & $31(5)$ \\
\hline Belardinelli 1995 & 55 & Stable CHF, IHD or IDCM, NYHA II/III & $27(4)$ \\
\hline Hambrecht 1995 & 22 & Stable CHF, IHD or IDCM, NYHA II/III & $26(9)$ \\
\hline Killavuori 1995 & 20 & Stable CHF, IHD or IDCM, NYHA II/III & $24(6)$ \\
\hline Killavuori 1995 & 27 & Stable CHF, IHD or IDCM, NYHA II/III & $24(5)$ \\
\hline Meyer 1996 & 18 & Stable CHF, IHD or IDCM, NYHA II/III & $21(1)$ \\
\hline Meyer 1996 & 16 & Stable CHF, IHD or IDCM, NYHA II/III & $23(3.2)$ \\
\hline Piepoli 1996 & 12 & Stable CHF, NYHA II/III & $26.4(1.6)$ \\
\hline Magnusson 1996 & 11 & Stable CHF, IHD or IDCM, NYHA II/IV & $19(11)$ \\
\hline Hambrecht 1997 & 18 & Stable CHF, IDCM, NYHA II/III & $34(5)$ \\
\hline ELVD study, Giannuzzi 1997 & 77 & 3-4 weeks after first MI, no history of heart failure & $34(5)$ \\
\hline Willenheimer 1998 & 49 & Stable CHF, IHD or IDCM, $\geqslant 8$ "Boston points" & $35(11)$ \\
\hline Belardinelli 1999 & 99 & Stable CHF, IHD or IDCM, NYHA II/III & $28(5)$ \\
\hline CHANGE study, Wilenga 1999 & 80 & Stable CHF, IHD or IDCM, NYHA II/III & $26(10)$ \\
\hline Hambrecht 2000 & 73 & Stable CHF, IHD or IDCM, NYHA II/III & $27(9)$ \\
\hline ELVD-CHF just completed, Giannuzzi & 86 & Stable CHF, IHD or IDCM, NYHA II/III & $23(4)$ \\
\hline
\end{tabular}

${ }^{\star}$ Mean $(\mathrm{SD})$

CHF, congestive heart failure; IHD, ischaemic heart disease; IDCM, idiopathic dilated cardiomyopathy; MI, myocardial infarction; NYHA, New York Heart Association.

\section{Clinical experience on exercise training in heart failure}

After the pioneering work of Sullivan and colleagues ${ }^{14}$ and the seminal small controlled study of Coats and associates, ${ }^{15}$ there have been a number of small studies investigating the benefits of exercise training in chronic heart failure. Table 1 presents the main characteristics of these investigations. Patients in these studies were generally in New York Heart Association (NYHA) functional class II and III, had been stable for the previous three months, had a left ventricular ejection fraction lower than $40 \%$, and were in sinus rhythm. Control group patients were required to maintain their routine daily activity and standard medication. Exercise intensity varied from $50-80 \%$ of maximum using a cycle, rowing, walking, swimming or ball games. Sessions lasted from 30-60 minutes, performed 3-5 times per week. The duration of the programmes varied from 1-6 months. Some of the programmes included a calisthenic component.

Significant increases in peak oxygen consumption $\left(\mathrm{Vo}_{2}\right)$, workload or exercise duration were reported in most studies. Most studies also reported significant increases in $\mathrm{Vo}_{2}$ around the anaerobic threshold as determined by ventilatory and lactate indices. On the whole physical training and angiotensin converting enzyme (ACE) inhibitor treatment have been shown to improve physical performance similarly in patients with heart failure - an increase in peak $\mathrm{VO}_{2}$ of around $2 \mathrm{ml} / \mathrm{kg} / \mathrm{min}$ and in exercise time of about 1.5 minutes.

Many patients with advanced heart failure are severely limited by symptoms of fatigue and/or dyspnoea, and the performance and maintenance of a training programme can be problematic. In fact, in 16 stable patients with moderate to severe heart failure, ejection fraction $<30 \%$ and peak $\mathrm{VO}_{2}<15 \mathrm{ml} / \mathrm{kg} / \mathrm{min}$, in NYHA functional class II/III and receiving optimal treatment, Opasich and colleagues ${ }^{16}$ observed at rest higher leg oxygen consumption, higher arteriovenous $\mathrm{O}_{2}$ difference $(7.2 v 5.4 \mathrm{ml} \%)$ and a lower femoral vein $\mathrm{pH}$ than in controls. At $20 \mathrm{~W}$, while the leg flow and the leg $\mathrm{O}_{2}$ consumption were similar in heart failure patients and in controls, the lactate release was greatly increased as was the noradrenaline (norepinephrine) release in heart failure patients. During the recovery period, the oxygen debt was inversely related to the exercise capacity. Thus, the efficiency of muscle metabolic processes is impaired in stable patients with severe heart failure during physical exercise mimicking light daily life activity. For this reason the investigation on low intensity exercise programmes is of particular interest in heart failure patients. Recently, Belardinelli and colleagues ${ }^{17}$ documented a $17 \%$ increase in peak $\mathrm{VO}_{2}$ with low intensity training for eight weeks in patients with heart failure. Interestingly, a very close correlation was noted between changes in venous lactate and both adrenaline (epinephrine) and noradrenaline after training in these patients. Moreover correlations were found between changes in volume density of mitochondria and changes in both peak oxygen uptake and lactate threshold. Pronounced benefits were also obtained by Demopoulos and colleagues in 16 patients with severe heart failure trained at low workload for one hour a day, four times a week for 12 weeks. ${ }^{18}$ Peak $\mathrm{VO}_{2}$ and calf and forearm reactive hyperaemia were measured before and during training. Peak $\mathrm{VO}_{2}$ increased from 11 to $14 \mathrm{ml} / \mathrm{kg} / \mathrm{min}$ after six weeks of training, and peak reactive hyperaemia in trained muscles also increased. These increases in calf peak reactive hypaeremia and peak $\mathrm{VO}_{2}$ were linearly related. Thus, low intensity exercise programmes performed at about $50 \%$ of peak exercise capacity have been shown to be effective in patients with heart failure.

To date there have been no reports of serious problems related to exercise training in heart failure patients. This is partly because of the exclusion of patients who have evidence of significant arrhythmias or abnormal blood pressure responses detected during laboratory based exercise testing, but also attests to the general safety of the exercise training programmes.

\section{Does the heart benefit from physical training?}

The investigations on the effects of physical training in patients with left ventricular dysfunction or heart failure mainly focused on the peripheral effects of exercise. At best, no changes were observed in the heart and central haemodynamics.

However a few years ago, Judgut and colleagues ${ }^{19}$ reported a significant deterioration in both global and regional left ventricular function after 12 weeks of exercise 
training in a small group of patients with anterior myocardial infarction. In trained patients with anterior infarct an increase of peak shape distortion index was evident after training. The authors concluded that physical training might be injurious in patients with an extensive transmural infarct. This study had considerable impact on clinical practice, raising a cautionary note that the potential to alter global ventricular size and topography adversely by physical training exists in patients with anterior infarction. However, this study had several limitations. It was carried out on small groups of patients and was not designed as a randomised clinical trial. Standardisation of timing of exercise training was lacking and the topographic indices were derived only from echocardiographic short axis views. More recently the group of Le Yemtel, ${ }^{18}$ from New York, observed that left ventricular enlargement can occur in normal subjects and in patients with ischaemic heart disease after physical training programmes lasting more than three months and performed at workloads corresponding to about $70 \%$ of the peak $\mathrm{Vo}_{2}$; they argued that conventional training programmes may be harmful in patients with heart failure because the ventricles would be exposed to periodic increases in wall stress which might represent a stimulus for progressive left ventricular enlargement. Such reasonable cautionary considerations required a direct verification of what happens to the heart during long term physical training. In fact, the short term improvement in physical performance usually reported in the small trials available in the literature dealing with physical training in heart failure patients is not enough to rule out the possibility of silent, unfavourable progression of ventricular remodelling.

Accordingly, a series of multicentre randomised controlled trials were performed in Italy to assess the effects of long term exercise training programmes on several end points, including global ventricular cavity dimension, function, and topography. Several secondary aims were defined, including among others the role of myocardial ischaemia in the possible deterioration of left ventricular function, and changes in autonomic nervous system balance related to physical training.

The first study performed was the EAMI (exercise in anterior myocardial infarction) trial, ${ }^{20}$ in which 95 patients with anterior myocardial infarction were randomised to six months physical training versus usual life style, 1-2 months after the acute event. The results showed no significant deterioration of left ventricular function after training.

The second study was the ELVD (exercise in left ventricular dysfunction) trial. ${ }^{21}$ In this study 77 patients with ejection fraction $<40 \%$ after a first Q wave myocardial infarction were randomly assigned to a six month exercise training programme or control. Seventy eight per cent of patients were given ACE inhibitors and $47 \% \beta$ blockers. At the end of the training programme work capacity increased by $20 \%$, and the rate-pressure product at the same submaximal workload decreased by $15 \%$ in trained patients and not in controls. Moreover, in the control group both left ventricular end diastolic and end systolic volumes increased with an unchanged ejection fraction while in trained patients end diastolic volume did not change, and end systolic volume decreased with a resulting increase in ejection fraction. The extent of left ventricular wall motion asynergy decreased in the trained group but not in the control group, whereas regional dilatation increased in the controls and not in the trained group. Similarly, Dubach and colleagues, ${ }^{22}$ using magnetic resonance imaging, reported no detrimental effect on wall thickness and thinning of both infarcted and non-infarcted regions after a high intensity two month exercise training programme in postinfarct patients with left ventricular dysfunction. These findings confirmed that, in postinfarction patients with poor left ventricular function, long term exercise training does not impair left ventricular function and topography, may attenuate the unfavourable remodelling response, and possibly improves both global and regional function over time.

A third multicentred, randomised trial, the ELVD$\mathrm{CHF},{ }^{23}$ was designed to investigate the effects of a moderate intensity six month training programme on work capacity, left ventricular function, and quality of life in patients with chronic heart failure and low work capacity. Eighty six patients with a history of heart failure of any aetiology, clinically stable over the previous three months, with ejection fraction $<40 \%$ and a work capacity $<20 \mathrm{ml} /$ $\mathrm{kg} / \mathrm{min} \mathrm{O}_{2}$ consumption, were enrolled and randomised to a six month training programme or usual life. Their mean age was 60 years; two third of the patients had an ischaemic cardiomyopathy, two thirds were in NYHA functional class II, and one third were in class III. The patients were receiving optimal medical treatment $(92 \%$ were given ACE inhibitors and $20 \% \beta$ blockers). Maximal work capacity, as well as the six minute walking distance, increased by about $20 \%$ in trained patients whereas they did not change in controls. The anaerobic threshold also increased in trained subjects but not in the controls. Importantly, the same features of cardiac dynamics observed in the ELVD 1 study in asymptomatic patients with poor left ventricular function were also found in the ELVD-CHF study in symptomatic patients with heart failure. A progression in remodelling of the left ventricle was noted in the control group with increases in both end diastolic and end systolic volumes, while the training group showed an opposite trend with decreases in both end diastolic and end systolic volumes associated with an increase in ejection fraction. Very recently, similar results have been reported by Hambrecht and colleagues, ${ }^{24}$ who showed that long term exercise training in patients with stable chronic heart failure is associated with reduction of peripheral resistance and results in small but significant improvements in stroke volume, ejection fraction, and reduction in left ventricular dimension.

Thus, on the whole the reported studies showed that asymptomatic patients with left ventricular dysfunction and patients with heart failure undergoing regular exercise while receiving optimal medical treatment may well derive additional symptomatic and functional benefits from physical training, with no impairment of ventricular function. However, further studies are warranted to establish clearly the long term effect of exercise training on left ventricular systolic function and cardiomegaly.

\section{Why can exercise training be beneficial in heart failure?}

There is clear evidence that exercise programmes produce a training effect in heart failure with improvements in symptoms of fatigue and dyspnoea. The question is why? The benefits appear to be primarily related to changes in peripheral haemodynamics and skeletal muscle by reversing a "deconditioning" process, common in sedentary heart failure patients. Improvements in peripheral haemodynamics and in the oxidative capacity of skeletal muscle have been demonstrated. Leg blood flow has been shown to increase at maximal exercise while during submaximal exercise significant decreases in blood lactate concentrations and vascular resistance occur in trained patients. ${ }^{142526}$ Increase in mitochondria and improvements in oxidative metabolism have been demonstrated by several techniques including nuclear magnetic resonance spectroscopy. ${ }^{6-822} 2425$

As mentioned above, patients with heart failure exhibit a blunted vasodilatory capacity in the skeletal muscles 
during exercise, which contributes to increased total peripheral resistances and peripheral hypoperfusion with a subnormal blood flow response to exercise. Two primary reasons for this reduction in nutritive blood flow are a decreased release of endothelium derived relaxing factor (nitric oxide vasodilator system) from arterioles supplying the metabolic active muscles, and an exaggerated increase in vasoconstrictive neurohormones such as endothelin, noradrenaline, renin, angiotensin II, and vasopressin (vasoconstrictor system). Although not exhaustively studied in heart failure, there is evidence that regular physical exercise can improve endothelial dysfunction, reduce peripheral vascular resistances, and increase skeletal muscle blood flow. ${ }^{1426}{ }^{25}$ Whether this is predominantly obtained by reducing vasoconstrictor tone, by improving large vessel function, or by enhancing endothelial vasodilator function is not known. It has been recently shown that exercise corrects endothelium dependent vasodilation of the skeletal muscle vasculature after stimulation with acetylcholine and even improves basal endothelial nitric oxide formation. ${ }^{24}$ Changes in endothelial function of the skeletal musculature of the lower limb were related to changes in peripheral resistance. This observation is consistent with the hypothesis that exercise therapy exerts its primary effects on the endothelial function of peripheral resistance vessels, contributing to the reduction in peripheral resistances at rest and at peak exercise. Peripheral vascular resistance may also be reduced by attenuating the sympathetic overactivity and by increasing vagal tone, as noted after exercise training in healthy subjects and in heart failure patients. ${ }^{27}$ In the recent study by Hambrecht and colleagues, ${ }^{24}$ however, changes in plasma catecholamine concentrations were not correlated with changes in peripheral resistances, indicating that training induced afterload reduction is not solely or primarily caused by reduced plasma catecholamines.

As previously mentioned, the ventilatory response to exercise is exaggerated in heart failure patients. The minute ventilation/carbon dioxide production $\left(\mathrm{VE} / \mathrm{VCO}_{2}\right)$ slope has been reported to be an independent prognostic indicator for patients with heart failure. ${ }^{28}$ Peak $\mathrm{VO}_{2}$ has also been reported to have important prognostic value in chronic heart failure patients. Training can significantly reduce minute ventilation during submaximal workloads and also reduce the regression slope relating minute ventilation to carbon dioxide production $\left(\mathrm{VE} / \mathrm{VCO}_{2}\right.$ slope) at submaximal and maximal workloads. ${ }^{27} 2930$

Other prognostic indicators are abnormal heart rate variability and increased concentrations of plasma noradrenaline. In a multicentred study the European heart failure training group reported that resting concentrations of noradrenaline and adrenaline decreased after training, indicating amelioration of the adrenergic overactivation that is commonly found in heart failure patients. ${ }^{31}$ This and other studies have also reported improvements in both the heart rate variability and high and low frequency fluctuations of the R-R interval. Thus training has been shown to alter autonomic balance favourably by ameliorating adrenergic overactivation and increasing parasympathetic drive.

Such changes in neurohumoral factors, and ventilatory and heart rates would allow the same work to be achieved at a lower level of metabolic cost, and reduce the unfavourable ventricular remodelling process.

\section{Exercise training: how and for whom?}

How to get exercise training in clinical practice? Despite the increased attention given to using moderate exercise training in the treatment plan of patients with stable heart failure, few guidelines exist relative to prescribing exercise to these patients. Recently the working group on exercise testing and exercise physiology and the working group on heart failure of the European Society of Cardiology released the recommendations on exercise testing and training in heart failure, summing up what is known in this field and suggesting how to translate this knowledge into practical prescription. ${ }^{32}$

Does this mean that there is evidence enough to incorporate exercise training as a therapeutic measure for the universe of heart failure patients? The answer is no. The available experience relates to selected patients; the trials performed were small, often involving just one centre, and with design problems in part inherent to the open nature of the experiments, in part related to the comprehensive approach to the treated patients, which makes it difficult to evaluate the effect of exercise in subjects exposed to multiple interventions. The study group which produced the European recommendations ${ }^{32}$ highlighted a number of unanswered questions, including, among others, whether the training effects could be maintained over the long term, whether exercise training is practical in multiple medical settings outside of enthusiastic and well motivated specialist clinics, whether mortality and morbidity can be affected by a large prescription of exercise training, and whether this strategy would be cost effective.

A large, properly designed trial aimed at assessing mortality and morbidity in a (reasonably) unselected population of heart failure patients is warranted. The initiative to organise such a trial has been taken by the clinical trials group at the Royal Brompton Hospital in London. As usually occurs when an object of research is not profitable, thereby not supported by commercial companies, the problem of funding becomes almost insoluble. However, the clinical need is there. ${ }^{33}{ }^{34}$ The working groups on heart failure and on cardiac rehabilitation and exercise physiology of the European Society of Cardiology strongly support a joint effort of the cardiological community to provide definitive evidence in such an important area of a comprehensive approach to heart failure. Let us hope that even improbable events can sometimes happen.

L TAVAZZI

IRCCS Policlinico S Matteo,

Cardiology Department,

P. le Golgi 2,

27100 Pavia, Italy

l.tavazzi@smatteo.pv.it

IRCCS Fondazione S Maugeri,

Cardiology Department,

Veruno, Italy

1 Rowell LB, O'Leary DS. Reflex control of the circulation during exercise: chemoreflexes and mechanoreflexes. F Appl Physiol 1990;69:407-18.

2 Saltin B, Radegran G, Koskolou MD, et al. Skeletal muscle blood flow in humans and its regulation during exercise. Acta Physiol Scandinavica 1998; 162:421-36.

3 Sullivan MJ, Knight D, Higginbotham MB, et al. Relation between central and peripheral hemodynamics during exercise in patients with chronic heart failure. Muscle blood flow is reduced with maintenance of arterial heart failure. Muscle blood flow is reduced with

perfusion pressure. Circulation 1989,80:769-81.

4 Wilson JR, Martin JL, Schwartz D et al. Exercise intolerance in patients with chronic heart failure: Role of impaired nutritive flow to skeletal muscle. Circulation 1984;69:1079-87.

5 Drexler H,Hayoz D, Munzel T, et al. Endothelial function in congestive heart failure. Am Heart $\mathcal{F}$ 1993;126:761-4.

6 Minotti JR, Johnson EC, Hudson TH, et al. Skeletal muscle response to exercise training in congestive heart failure. $\mathcal{F}$ Clin Invest 1990;86:751-8.

7 Adamopoulos S, Coats AJ, Brunotte F, et al. Physical training improves skeletal muscle metabolism in patients with chronic heart failure. $7 \mathrm{Am}$ Coll Cardiol 1993;21:1101-6.

8 Hambrecht R, Fiehn E, Yu JT, et al. Effects of endurance training on mitochondrial ultrastructure and fiber type distribution in skeletal muscle of patients with stable chronic heart failure. $\mathcal{F}$ Am Coll Cardiol 1997;29:106773 .

9 Meyer K, Westbrook S, Schwaibold M, et al. Cardiopulmonary determinants of functional capacity in patients with chronic heart failure compared with normals. Clin Cardiol 1996;19:944-8.

10 Sullivan MJ, Higginbothan MB, Cobb FR. Increased exercise ventilation in patients with chronic heart failure: Intact ventilatory control despite hemodynamic and pulmonary abnormalities. Circulation 1988;77:552-9. 
11 Mancini D, Henon D, La Manca J, et al. Benefit of selective respiratory muscle training on exercise capacity in patients with chronic congestive

12 Coats AJS, Clark AI, Piepoli M, et al. Symptoms and quality of life in heart failure : the muscle hypothesis. Br Heart f 1994;72:S36-9.

13 Piepoli M, Clark AL, Volterrani $M$, et al. Contribution of muscle afferents to the hemodynamic, autonomic, and ventilatory responses to exercise in patients with chronic heart failure: effects of physical training. Circulation 1966;93:940-52.

14 Sullivan MJ, Higginbotham MB, Cobb FR. Exercise training in patients with severe left ventricular dysfunction: hemodynamic and metabolic effects. Circulation 1988;78:506-15.

15 Coats AJS, Adamopoulos S, Meyer TE, et al. Effects of physical training in chronic heart failure. Lancet 1990;335:63-6.

16 Opasich C, Pasini E, Aquilani R, et al. Skeletal muscle function at low level as a model for daily activities in patients with chronic heart failure. Eur Heart f 1997;18:1626-31.

17 Belardinelli R, Georgiou D, Scocco V, et al. Low intensity exercise training in patients with chronic heart failure. $\mathcal{F}$ Am Coll Cardiol 1995;26:975-82.

18 Demopoulos L, Bijou R, Fergus I, et al. Exercise training in patients with severe congestive heart failure: enhancing peak aerobic capacity while severe congestive heart failure: enhancing peak aerobic capacity while
minimizing the increase in ventricular wall stress. $7 \mathrm{Am}$ Coll Cardiol 1997; minimizing

19 Jugdutt BI, Michorowski BL, Kappagoda CT. Exercise training after anterior $\mathrm{Q}$ wave myocardial infarction: importance of regional function and topography. $\mathcal{f}$ Am Coll Cardiol 1988;12:362-72.

20 Giannuzzi P, Tavazzi L, Temporelli PL, et al. Long-term physical training and left ventricular remodeling after anterior myocardial infarction: results of the exercise in anterior myocardial infarction (EAMI) study group. $\mathcal{F} \mathrm{Am}$ Coll Cardiol 1993;22:1821-9.

21 Giannuzzi P, Temporelli PL, Corrà U, et al. Attenuation of unfavorable remodeling by exercise training in postinfarction patients with left ventricular dysfunction: results of the exercise in left ventricular dysfunction (ELVD) trial. Circulation 1997;96:1790-7.

22 Dubach P, Myers J, Dziekan G, et al. Effect of exercise training on myocardial remodeling in patients with reduced left ventricular function after myocardial infarction. Circulation 1997;96:2060-7.
23 Giannuzzi $\mathrm{P}$, Temporelli $\mathrm{Pl}$, Corrà U, et al . Long-term exercise training in patients with chronic heart failure: results of the ELVD-CHF trial. Circulation 1997;96:I-771.

24 Hambrecht R, Gielen S. Linke A, et al. Effects of exercise training on left ventricular function and peripheral resistance in patients with chronic heart failure: a randomized trial. $7 A M A$ 2000;283:3095-101.

25 Hambrecht R, Fiehn E, Weigl C, et al. Regular physical exercise corrects endothelial dysfunction and improves exercise capacity in patients with chronic heart failure. Circulation 1998;98:2709-15.

26 Horning B, Maier V, Drexler H. Physical training improves endothelial function in patients with chronic heart failure. Circulation 1996;93:210-14.

27 Coats AJS, Adamopoulos S, Radaelli A, et al. Controlled trial of physical training in chronic heart failure: exercise performance, hemodynamics, ventilation, and autonomic function. Circulation 1992;85:2119-31.

28 Kleber FX, Vietzke G, Werecke KD, et al. Impairment of ventilatory efficiency in heart failure. Circulation 2000;101:2803-9.

29 Meyer K, Schwaibold M, Westbrook S, et al. Effects of short-term exercise training and activity restriction on functional capacity in patients with severe chronic congestive heart failure. Am f Cardiol 1996;78:1017-22.

30 Kiilavuory K, Sovijarvi A, Naveri $\mathrm{H}$, et al. Effect of physical training on exercise capacity and gas exchange in patients with chronic heart failure. Chest 1999;110:985-91.

31 European Heart Failure Training Group. Experience from controlled trials of physical training in chronic heart failure: protocol and patient factors in effectiveness in the improvement in exercise tolerance. Eur Heart $\mathcal{f}$ 1998;19:466-75.

32 Working Group on Cardiac Rehabilitation and Exercise Physiology and Working Group on Heart Failure of the European Society of Cardiology. Recommendations for exercise training in chronic heart failure patients. Eur Heart $\mathcal{F}$ 2001;22:125-35.

33 Villani GQ, Capucci A, Piepoli MS. Emerging concepts in exercise training in chronic heart failure. Ital Heart 7 2000;1:795-800.

34 Dickstein $\mathrm{K}$. Exercise training in heart failure: time to go beyond surrogate endpoints. Eur f Heart Failure 2000;2:5-6.

\section{IMAGES IN CARDIOLOGY}

\section{Self portrait of patient with severe cardiac pain awaiting surgery}

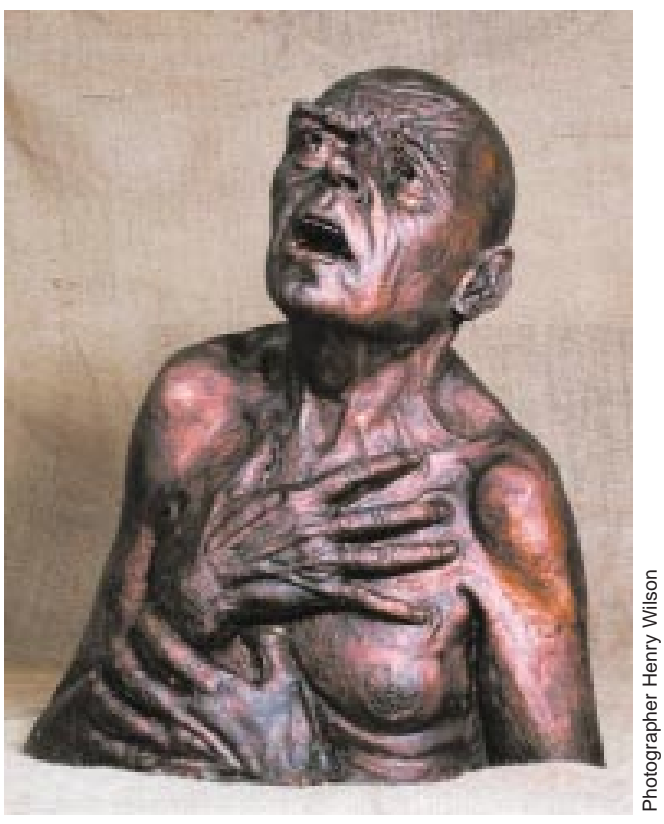

This sculpture is a self portrait. It was created by a patient who was having severe pain as a result of an aneurysm of the ascending aorta. It expresses the sensation he experienced while waiting for surgery. The patient underwent a successful operation and has since recovered well. $\mathrm{He}$ is now training to be a nurse.

ROGER HALL

Editor 